\title{
Worldwide conservation hotspots for Soricomorpha focusing on endemic island taxa: an analysis at two taxonomic levels
}

\author{
Giovanni Amori ${ }^{1, *}$, Federica Chiozza $^{2}$, Carlo Rondinini ${ }^{2}{ }^{2}$ Luca Luiselli $^{3}$ \\ ${ }^{1}$ CNR-Institute of Ecosystem Studies, Viale dell'Università 32, 00185 Rome, Italy \\ ${ }^{2}$ Department of Biology and Biotechnology, Sapienza Università di Roma, Viale dell'Università 32, 00185 Roma, Italy \\ ${ }^{3}$ Centre of Environmental Studies Demetra, Eni Environmental Department, via Olona 7, 00198 Rome, Italy
}

\begin{abstract}
Identifying conservation priorities is crucial in the modern world, and biodiversity hotspots have been vital instruments in better defining the main areas requiring conservation. This paper analyzes distribution patterns of threatened Soricomorpha genera and species to evaluate whether the current biodiversity hotspots network really covers these taxa. We also analyze the Soricomorpha species that are endemic to islands worldwide in order to define the main islands in terms of both endemic species richness and threatened species richness. At the genus level, all threatened taxa are represented within hotspots, whereas $18 \%$ of the threatened species (particularly in the Afrotropics) do not occur in any biodiversity hotspot. Approximately $35 \%$ of island endemic species are threatened, particularly in the Oriental region. Most of the threatened endemics are found within forest habitats. Both mainland (Cameroon and Congo) and island (particularly Andamans, Sri Lanka, Bioko) forest ecosystems deserve the particular attention of conservation organizations. In addition, basic research on the ecology, taxonomy, and distribution of threatened Soricomorpha species (particularly in the tropical regions) is urgently needed.
\end{abstract}

KEY WORDS: Hotspots · Soricomorpha $\cdot$ Conservation · Endemicity · Islands

\section{INTRODUCTION}

Identifying conservation priorities is crucial in the modern world where human activities have a strong impact on the natural environment and resources (Wilson 1992, Myers 1998). An important dilemma for conservationists has hence been to define which areas are the most immediately important for conserving biodiversity (Wilson 1992, Myers et al. 2000, Mittermeier et al. 2004). Brooks et al. (2006) identified 9 major institutional templates of global biodiversity conservation prioritization, with most of these templates prioritizing highly irreplaceable regions: some are reactive (prioritizing high vulnerability) and others are proactive (prioritizing low vulnerability). Some proposals for systematic conservation of priority areas are based on biogeographic units, which are typically defined a priori by specialist perception of the distribution of biodiversity (Brooks et al. 2006), as, for example, the ecoregions (Olson \& Dinerstein 1998).

One of the 9 templates advised by Brooks et al. (2006) is the biodiversity hotspots approach (Myers et al. 2000, Stuart et al. 2004). This approach defines 34 'biodiversity hotspots, which hold especially high numbers of endemic species and which together cover $\sim 2.3 \%$ of the Earth's land surface (Stuart et al. 2004). When biodiversity hotspots take endemic spe- 
cies richness into consideration, the emerging patterns can be extremely useful because, although it has been demonstrated that the correlation between global richness and endemism is low, aggregating regions into appropriate hotspots selected for high levels of endemism captures far more species than single unaggregated regions (Lamoreux et al. 2006). Each hotspot faces extreme threats and has already lost at least $70 \%$ of its original natural vegetation (Myers et al. 2000). Over $50 \%$ of the world's plant species and $42 \%$ of all terrestrial vertebrate species are endemic to the 34 biodiversity hotspots (Myers et al. 2000, Stuart et al. 2004). Nonetheless, biodiversity hotspots have also been criticized for several reasons, including the fact that they do not consider phylogenetic diversity and/or do not give adequate representation of taxa other than vascular plants (Kareiva \& Marvier 2003).

A majority of endemic taxa around the world are found on islands, where they are particularly vulnerable to changes in biotic factors (Sala et al. 2000). Although the general link between endemicity rate, island ecosystems and threatened status is widely recognized by the international community of conservationists, there is still very scarce quantitative information for many animal groups, including small mammals (Amori et al. 2008). This is particularly unfortunate if we consider that small mammals (rodents and insectivores) account for $50 \%$ of the total number of mammal species $(\mathrm{n}=$ 5416), with 2277 rodent and 428 insectivore species (Wilson \& Reeder 2005). In addition, many of these species are threatened with extinction (Amori et al. 2011a).

Another important point to be considered is that identification of biodiversity hotspots was based on taxa other than small mammals, particularly insectivores (Myers et al. 2000, Stuart et al. 2004). Hence, it is interesting to evaluate how small mammals may fit with the already defined hotspots and how they can contribute towards emphasizing the relevance of the various hotspots for biodiversity conservation.

In this paper, we analyze patterns of distribution across zoogeographic regions of threatened Soricomorpha species in order to evaluate whether the current biodiversity hotspots network really covers this taxon. We also focus our attention on the species that are endemic to islands. In particular, we define the main islands in terms of endemic species and threatened species richness. Our data are aimed at international relevance for better defining global conservation strategies for soricomorphs, particularly since these species are rarely considered in conservation management, being noncharismatic and usually scarcely studied in the field (Amori \& Gippoliti 2000).

\section{MATERIALS AND METHODS}

We extracted data for insectivores from the International Union for Conservation of Nature (IUCN) Red List presence record (Soricomorpha according to Wilson \& Reeder 2005; available at www.iucn. redlist.org) occurring throughout the world. We excluded from the analysis the allochtonous species.

Biodiversity hotspots were selected according to Myers et al. (2000) and Stuart et al. (2004); threatened soricomorph diversity at the genus level was evaluated according to the criterion of Amori \& Gippoliti (2000), where a threatened genus was that for which all extant species were listed as Critically Endangered, Endangered or Vulnerable by the IUCN (2010). For threatened species, we used the IUCN lists (2010). Soricomorph nomenclature followed Wilson \& Reeder (2005). In this regard, it is necessary to consider that soricomorphs do not have a global distribution, being absent from Australia, Papua-New Guinea and South America. Hence, our study deals only with the biodiversity hotspots situated outside these major regions (Churchfield 1990).

For each zoogeographical region (i.e. Nearctic, Neotropical [excluding South America], Palearctic, Afrotropical, Oriental, and Australian [excluding Australia and Papua-New Guinea; henceforth Australasian]), we considered whether the distribution range of each threatened genus/species matched, at least partially, with one or more of the biodiversity hotspots. We assessed the overlap between threatened taxa distribution and biodiversity hotspots using the species distribution polygons (GIS shapefiles) available in IUCN (2010) using ArcGIS software. If at least $5 \%$ of a species' range overlapped with any biodiversity hotspot, we considered that the taxon was covered by that biodiversity hotspot; otherwise, the threatened taxon was considered disjunct from hotspots. Chi-squared tests were used to explore whether the frequencies of threatened taxa within and outside of biodiversity hotspots differed significantly either globally or at a zoogeographical region level. Alpha was assessed at 5\%. Statistics were performed using STATISTICA v6.0 software. Chi-square tests were calculated using the observedversus-expected option as available in STATISTICA. 


\section{RESULTS}

\section{Distribution of threatened taxa within and outside of biodiversity hotspots}

The distribution of species richness of soricomorphs throughout the world revealed peaks in the Central African rainforest and Southeast Asia (Fig. 1). Overall, 9 out of 45 soricomorph genera $(20 \%)$ were threatened worldwide, all of which were found within biodiversity hotspots (Table 1). Of the 45 genera, $17(37.7 \%)$ had at least 1 threatened species. Of 428 soricomorph species, 81 $(18.9 \%)$ were threatened; of these $14(17.3 \%)$ did not occur within biodiversity hotspots, and 67

Table 1. Threatened soricomorph genera included within biodiversity hotspots as defined by Myers et al. (2000) and Stuart et al. (2004)

\begin{tabular}{|c|c|c|}
\hline Genus & Distribution & $\begin{array}{l}\text { Zoogeographical } \\
\text { region }\end{array}$ \\
\hline Desmana & $\begin{array}{c}\text { Russia, Ukraine, } \\
\text { Kazakhstan }\end{array}$ & Palearctic \\
\hline Feroculus & Sri Lanka & Oriental \\
\hline Galemys & Spain, France, Portugal & Palearctic \\
\hline Neohylomys & Hainan Island (China) & Oriental \\
\hline Podogymnura & Philippines & Oriental \\
\hline Ruwenzorisorex & $\begin{array}{l}\text { Burundi, Rwanda, } \\
\text { Democratic Republic } \\
\text { of Congo, Uganda }\end{array}$ & Afrotropical \\
\hline Solenodon & Cuba & Neotropical \\
\hline Solisorex & Sri Lanka & Oriental \\
\hline Surdisorex & Kenya & Afrotropical \\
\hline
\end{tabular}

$(82.7 \%)$ did. Hence, among the threatened species occurring throughout the world, the proportion of those occurring within hotspots was significantly higher than those occurring outside of biodiversity hotspots $\left(\chi^{2}=34.68, \mathrm{df}=1, \mathrm{p}<0.00001\right)$. If we analyze the data by zoogeographic region (Table 2), a significantly higher proportion of threatened species $\left(\chi^{2}=43.3, \mathrm{df}=9, \mathrm{p}<0.0001\right)$ occurs within rather than outside of biodiversity hotspots (Fig. 2). However, there was considerable variation among regions: indeed, whilst nearly all threatened species are included in biodiversity hotspots in the Palearctic, Oriental and Neotropical regions, $\sim 30 \%$ of the Afrotropical threatened species are not found within biodiversity hotspots, particularly those occurring in the Congolese and southern Cameroonian forests (Fig. 2).

\section{Distribution of threatened taxa in relation to island endemicity}

Table 3 lists all species of soricomorphs endemic to islands, including their IUCN category. A total of 68 species are endemic to islands, with the Oriental regions housing the majority of them (Fig. 3a). The main islands in terms of number of endemic taxa were: Japan, the Philippines, Sri Lanka and Sulawesi (Fig. 3b). Of the endemic taxa occurring on these islands, $7.3 \%$ were Critically Endangered (CR), $22.1 \%$ Endangered (EN), and $5.9 \%$ Vulnerable (VU). Overall, $35.3 \%$ of the soricomorphs endemic to islands were threatened. The proportion of endan-



Fig. 1. Species richness of soricomorph species worldwide. Shading shows number of species known to occur in the respective regions 
Table 2. Threatened soricomorph species occurring in biodiversity hotspots (definition of hotspots follows Myers et al. 2000 and Stuart et al. 2004). DRC: Democratic Republic of Congo. CR: Critically Endangered; EN: Endangered; VU: Vulnerable; (?): presence uncertain

\begin{tabular}{|c|c|c|c|c|c|}
\hline Family & $\begin{array}{l}\text { IUCN } \\
\text { Status }\end{array}$ & Species & Distribution & $\begin{array}{l}\text { Zoogeographical } \\
\text { region }\end{array}$ & $\begin{array}{c}\text { Presence in } \\
\text { hotspots }\end{array}$ \\
\hline \multirow[t]{2}{*}{ Solenodontidae } & EN & Solenodon cubanus & Cuba & Neotropical & Yes \\
\hline & EN & Solenodon paradoxus & Hispaniola & Neotropical & Yes \\
\hline \multirow[t]{55}{*}{ Soricidae } & EN & Chimarrogale phaeura & Borneo & Oriental & Yes \\
\hline & $\mathrm{CR}$ & Congosorex phillipsorum & Tanzania & Afrotropical & Yes \\
\hline & VU & Crocidura allex & Kenya, Tanzania & Afrotropical & Yes \\
\hline & $\mathrm{CR}$ & Crocidura andamanens & Andaman Island & Oriental & Yes \\
\hline & EN & Crocidura ansellorum & Zambia, DCR, Angola & Afrotropical & No \\
\hline & EN & Crocidura baileyi & Ethiopia & Afrotropical & Yes \\
\hline & VU & Crocidura baluensis & Borneo & Oriental & Yes \\
\hline & EN & Crocidura bottegoide & Ethiopia & Afrotropical & Yes \\
\hline & EN & Crocidura canariensis & Canary Islands (Spain) & Palearctic & Yes \\
\hline & EN & Crocidura desperata & Tanzania & Afrotropical & Yes \\
\hline & VU & Crocidura eisentrauti & Cameroon & Afrotropical & No \\
\hline & VU & Crocidura fumosa & Kenya & Afrotropical & Yes \\
\hline & VU & Crocidura glassi & Ethiopia & Afrotropical & Yes \\
\hline & $\mathrm{CR}$ & Crocidura harenna & Ethiopia & Afrotropical & Yes \\
\hline & EN & Crocidura hikmiya & Sri Lanka & Oriental & Yes \\
\hline & VU & Crocidura hispida & Andaman Island & Oriental & Yes \\
\hline & $\mathrm{CR}$ & Crocidura jenkinsi & Andaman Island & Oriental & Yes \\
\hline & VU & Crocidura kivuana & DCR & Afrotropical & No \\
\hline & EN & Crocidura lanosa & DCR, Rwanda & Afrotropical & No \\
\hline & VU & Crocidura lucina & Ethiopia & Afrotropical & Yes \\
\hline & VU & Crocidura macmillani & Ethiopia & Afrotropical & Yes \\
\hline & VU & Crocidura manengubae & Cameroon & Afrotropical & Yes \\
\hline & EN & Crocidura miya & Sri Lanka & Oriental & Yes \\
\hline & EN & Crocidura negrina & Philippines & Oriental & Yes \\
\hline & $\mathrm{CR}$ & Crocidura nicobarica & Nicobar Islands & Oriental & Yes \\
\hline & VU & Crocidura orientalis & Java & Oriental & Yes \\
\hline & EN & Crocidura orii & Ryukyu Islands & Palearctic & Yes \\
\hline & EN & Crocidura phaeura & Ethiopia & Afrotropical & Yes \\
\hline & EN & Crocidura picea & Cameroon & Afrotropical & Yes \\
\hline & EN & Crocidura stenocepha & DCR, Uganda & Afrotropical & No \\
\hline & EN & Crocidura tansaniana & Tanzania & Afrotropical & Yes \\
\hline & EN & Crocidura tarella & DCR, Uganda & Afrotropical & No \\
\hline & EN & Crocidura telfordi & Tanzania & Afrotropical & Yes \\
\hline & EN & Crocidura thomensis & Sao Tome & Afrotropical & No \\
\hline & $\mathrm{CR}$ & Crocidura trichura & Christmas Island (Australia) & Oriental & Yes \\
\hline & EN & Crocidura usambarae & Tanzania & Afrotropical & Yes \\
\hline & $\mathrm{CR}$ & Crocidura wimmeri & Ivory Coast & Afrotropical & Yes \\
\hline & VU & Crocidura zimmermanni & Crete (Greece) & Palearctic & Yes \\
\hline & EN & Cryptotis endersi & Panama & Neotropical & Yes \\
\hline & VU & Cryptotis gracilis & Costa Rica & Neotropical & Yes \\
\hline & VU & Cryptotis griseoventris & Mexico, Guatemala & Neotropical & Yes \\
\hline & VU & Cryptotis magna & Mexico & Neotropical & Yes \\
\hline & EN & Cryptotis mera & Panama & Neotropical & Yes \\
\hline & $\mathrm{CR}$ & Cryptotis nelsoni & Mexico & Neotropical & Yes \\
\hline & VU & Cryptotis obscura & Mexico & Neotropical & Yes \\
\hline & VU & Cryptotis phillipsii & Mexico & Neotropical & Yes \\
\hline & EN & Feroculus feroculus & Sri Lanka & Oriental & Yes \\
\hline & EN & Myosorex blarina & DRC, Uganda & Afrotropical & No \\
\hline & $\mathrm{CR}$ & Myosorex eisentrauti & Bioko & Afrotropical & Yes \\
\hline & EN & Myosorex geata & Tanzania & Afrotropical & Yes \\
\hline & EN & Myosorex kihaulei & Tanzania & Afrotropical & Yes \\
\hline & VU & Myosorex longicaudatus & South Africa & Afrotropical & Yes \\
\hline & EN & Myosorex okuensis & Cameroon & Afrotropical & Yes \\
\hline & EN & Myosorex rumpii & Cameroon & Afrotropical & No \\
\hline & VU & Myosorex zinki & Tanzania & Afrotropical & Yes \\
\hline
\end{tabular}


Table 2 (continued)

\begin{tabular}{|c|c|c|c|c|c|}
\hline Family & $\begin{array}{l}\text { IUCN } \\
\text { Status }\end{array}$ & Species & Distribution & $\begin{array}{l}\text { Zoogeographical } \\
\text { region }\end{array}$ & $\begin{array}{c}\text { Presence in } \\
\text { hotspots }\end{array}$ \\
\hline \multirow[t]{21}{*}{ Soricidae } & VU & Notiosorex villai & Mexico & Neotropical & Yes \\
\hline & VU & Ruwenzorisorex sunco & Burundi, Rwanda, DRC, Uganda & Afrotropical & No \\
\hline & EN & Solisorex pearsoni & Sri Lanka & Oriental & Yes \\
\hline & VU & Sorex macrodon & Mexico & Neotropical & Yes \\
\hline & VU & Sorex milleri & Mexico & Neotropical & Yes \\
\hline & EN & Sorex pribilofensis & Saint Paul Island (Alaska) & Nearctic & No \\
\hline & $\mathrm{CR}$ & Sorex sclateri & Mexico & Neotropical & Yes \\
\hline & $\mathrm{CR}$ & Sorex stizodon & Mexico & Neotropical & Yes \\
\hline & $\mathrm{CR}$ & Suncus aequatorius & Kenya, (Tanzania?) & Afrotropical & Yes \\
\hline & EN & Suncus dayi & India & Oriental & No \\
\hline & EN & Suncus fellowesgordoni & Sri Lanka & Oriental & Yes \\
\hline & EN & Suncus mertensi & Flores (Indonesia) & Oriental & Yes \\
\hline & VU & Suncus montanus & India, Sri Lanka & Oriental & Yes \\
\hline & EN & Suncus zeylanicus & Sri Lanka & Oriental & Yes \\
\hline & VU & Surdisorex norae & Kenya & Afrotropical & Yes \\
\hline & VU & Surdisorex polulus & Kenya & Afrotropical & Yes \\
\hline & VU & Sylvisorex camerunensis & Cameroon, Nigeria & Afrotropical & Yes \\
\hline & EN & Sylvisorex howelli & Tanzania & Afrotropical & Yes \\
\hline & EN & Sylvisorex isabellae & Bioko & Afrotropical & Yes \\
\hline & VU & Sylvisorex lunaris & Burundi, Rwanda, DRC, Uganda & Afrotropical & No \\
\hline & EN & Sylvisorex morio & Cameroon & Afrotropical & No \\
\hline \multirow[t]{3}{*}{ Talpidae } & VU & Desmana moschata & Russia & Palearctic & Yes \\
\hline & VU & Galemys pyrenaicus & Spain, France, Portugal & Palearctic & Yes \\
\hline & EN & Mogera etigo & Japan & Palearctic & Yes \\
\hline
\end{tabular}

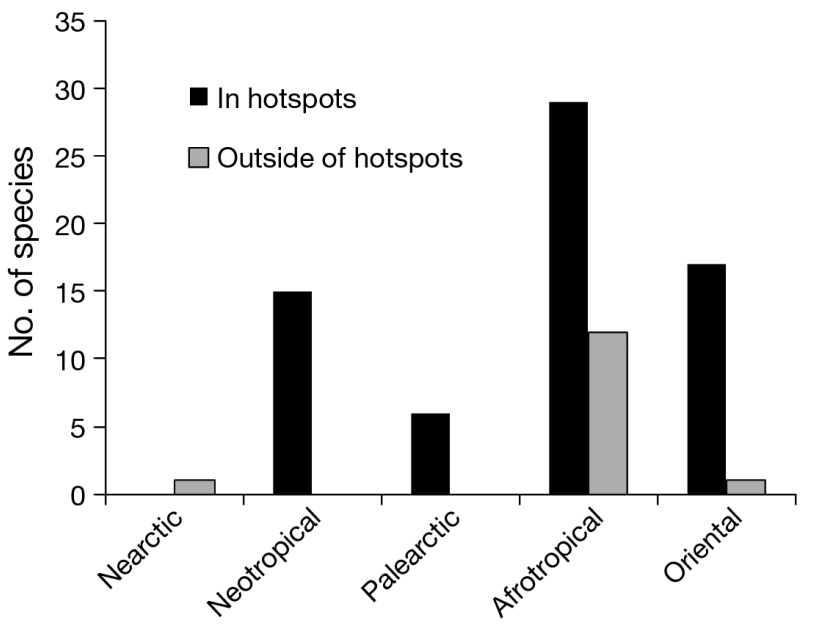

Fig. 2. Number of threatened soricomorphs species within and outside of biodiversity hotspots as defined by Myers et al. (2000) and Stuart et al. (2004) according to geographic regions

gered soricomorphs was significantly higher among species endemic to islands than among species nonendemic to islands ( $\chi^{2}$ test, $\left.\mathrm{df}=1, \mathrm{p}<0.01\right)$. The islands hosting the greatest number of endangered species were Andamans, Sri Lanka, and Bioko (Table 3).

\section{DISCUSSION}

At the genus level, this study confirms that biodiversity hotspots as currently defined (see Myers et al. 2000, Stuart et al. 2004) fully cover the diversity of threatened genera. This is important, of course, in terms of conservation, especially because other studies on small mammals showed a remarkable disjunction between threatened genera diversity and biodiversity hotspots (Amori et al. 2011b). In the case of rodents, however, most of the threatened genera found outside biodiversity hotspots occur in PapuaNew Guinea (Amori et al. 2011b), where soricomorphs are not present. On the other hand, $<1$ out of 5 threatened species are not represented in biodiversity hotspots. This mismatch between threatened species and biodiversity hotspots occurs in particular in the Afrotropical region (mainly Congo and Cameroon) where soricomorphs are often linked to rainforest habitats (Amori \& Luiselli 2011) that are currently suffering from severe fragmentation and exploitation (e.g. Davis \& Philips 2009), as for example, in the case of Sylvisorex spp. (Nowak 1999). We therefore suggest adding Congolese and Cameroonian forests to the list of biodiversity hotspots, at least for soricomorphs, in order to increase the effective- 
Table 3. Soricomorph species endemic to islands, including their distribution and IUCN status. LC: Least Concerned; DD: Data Deficient; Is.: Island(s). See Table 2 for other definitions

\begin{tabular}{|c|c|c|c|c|c|c|c|}
\hline Family & $\begin{array}{l}\text { IUCN } \\
\text { status }\end{array}$ & Species & Distribution & Family & $\begin{array}{l}\text { IUCN } \\
\text { status }\end{array}$ & Species & Distribution \\
\hline \multirow[t]{39}{*}{ Soricidae } & $\mathrm{LC}$ & Anourosorex yamashin & Taiwan & \multirow[t]{30}{*}{ Soricidae } & EN & \multirow{3}{*}{$\begin{array}{l}\text { Crocidura thomensis } \\
\text { Crocidura trichura }\end{array}$} & Sao Tome \\
\hline & EN & Chimarrogale phaeura & Borneo & & \multirow[t]{2}{*}{$\mathrm{CR}$} & & Christmas Is. \\
\hline & LC & Chimarrogale platycephala & Japan & & & & (Australia) \\
\hline & $\mathrm{DD}$ & Chimarrogale sumatrana & Sumatra & & \multirow[t]{2}{*}{$\mathrm{DD}$} & \multirow[t]{2}{*}{ Crocidura vosmaeri } & Bangka \\
\hline & $\mathrm{DD}$ & Chodsigoa sodalis & Taiwan & & & & (Indonesia) \\
\hline & $\mathrm{CR}$ & Crocidura andamanens & Andaman Is. & & LC & Crocidura watasei & Japan \\
\hline & VU & Crocidura baluensis & Borneo & & \multirow[t]{2}{*}{ VU } & \multirow{2}{*}{$\begin{array}{c}\text { Crocidura zimmermanni } \\
\text { (Greece) }\end{array}$} & \multirow[t]{2}{*}{ Crete } \\
\hline & $\mathrm{LC}$ & Crocidura beatus & Philippines & & & & \\
\hline & $\mathrm{LC}$ & Crocidura beccarii & Sumatra & & LC & Episoriculus fumidus & Taiwan \\
\hline & $\mathrm{LC}$ & Crocidura brunnea & Java, Bali & & EN & Feroculus feroculus & Sri Lanka \\
\hline & EN & Crocidura canariensis & Canary Is. & & $\mathrm{CR}$ & Myosorex eisentrauti & Bioko \\
\hline & & & (Spain) & & EN & Solisorex pearsoni & Sri Lanka \\
\hline & $\mathrm{LC}$ & Crocidura dsinezumi & Japan & & \multirow[t]{2}{*}{ LC } & \multirow[t]{2}{*}{ Sorex hosonoi } & Honshu \\
\hline & $\mathrm{LC}$ & Crocidura elongata & Sulawesi & & & & (Japan) \\
\hline & LC & Crocidura foetida & Borneo & & \multirow[t]{2}{*}{ LC } & \multirow{2}{*}{ Sorex jacksoni } & St Lauren Is. \\
\hline & DD & Crocidura grandis & Philippines & & & & (USA) \\
\hline & $\mathrm{LC}$ & Crocidura grayi & Philippines & & \multirow[t]{2}{*}{ DD } & \multirow[t]{2}{*}{ Sorex leucogaster } & Paramushir \\
\hline & EN & Crocidura hikmiya & Sri Lanka & & & & (Russia) \\
\hline & VU & Crocidura hispida & Andaman Is. & & \multirow[t]{2}{*}{ EN } & \multirow[t]{2}{*}{ Sorex pribilofensis } & Saint Paul Is. \\
\hline & $\mathrm{LC}$ & Crocidura hutanis & Sumatra & & & & (Alaska) \\
\hline & CR & Crocidura jenkinsi & Andaman Is. & & \multirow{2}{*}{$\begin{array}{l}\mathrm{LC} \\
\mathrm{DD}\end{array}$} & \multirow{2}{*}{$\begin{array}{l}\text { Sorex shinto } \\
\text { Suncus ater }\end{array}$} & Japan \\
\hline & LC & Crocidura lea & Sulawesi & & & & Borneo \\
\hline & $\mathrm{LC}$ & Crocidura lepidura & Sumatra & & \multirow{2}{*}{ EN } & \multirow{2}{*}{$\begin{array}{l}\text { Suncus fellowesgordoni } \\
\text { Suncus hosei }\end{array}$} & Sri Lanka \\
\hline & LC & Crocidura levicula & Sulawesi & & & & Borneo \\
\hline & $\mathrm{LC}$ & Crocidura maxi & Indonesia & & \multirow{2}{*}{ LC } & \multirow{4}{*}{$\begin{array}{c}\text { Suncus } \\
\text { madagascariensis } \\
\text { Suncus mertensi }\end{array}$} & Madagascar, \\
\hline & DD & Crocidura mindorus & Philippines & & & & Comoros \\
\hline & EN & Crocidura miya & Sri Lanka & & \multirow[t]{2}{*}{ EN } & & Flores \\
\hline & $\mathrm{DD}$ & Crocidura musseri & Sulawesi & & & & (Indonesia) \\
\hline & EN & Crocidura negrina & Philippines & & EN & \multirow{2}{*}{$\begin{array}{l}\text { Suncus zeylanicus } \\
\text { Sylvisorex isabellae }\end{array}$} & Sri Lanka \\
\hline & $\mathrm{CR}$ & Crocidura nicobarica & Nicobar Is. & & EN & & Bioko \\
\hline & $\begin{array}{l}\text { LC } \\
\text { VU }\end{array}$ & $\begin{array}{l}\text { Crocidura nigripes } \\
\text { Crocidura orientalis }\end{array}$ & $\begin{array}{l}\text { Sulawesi } \\
\text { Java }\end{array}$ & Solenodontidae & EN & Solenodon cubanus & Cuba \\
\hline & EN & Crocidura orii & Ryukyu Is. & & EN & Solenodon paradoxus & Hispaniola \\
\hline & LC & Crocidura palawanens & Philippines & Talpidae & $\mathrm{DD}$ & Euroscaptor mizura & Japan \\
\hline & $\mathrm{LC}$ & Crocidura paradoxura & Sumatra, & & LC & Mogera imaizumii & Japan \\
\hline & & & (Java?) & & NT & Mogera tokudae & Japan \\
\hline & $\mathrm{LC}$ & Crocidura rhoditis & Sulawesi & & $\mathrm{DD}$ & Mogera uchidai & Ryukyu Is. \\
\hline & LC & Crocidura sicula & Sicily & & LC & Mogera wogura & Japan \\
\hline & LC & Crocidura tanakae & Taiwan & & LC & Dymecodon pilirostris & Japan \\
\hline & $\mathrm{DD}$ & Crocidura tenuis & Timor & & LC & Urotrichus talpoides & Japan \\
\hline
\end{tabular}

ness of potential worldwide conservation of these neglected mammals.

Although our study revealed that the current biodiversity hotspots network covers nearly all the diversity of threatened soricomorph genera and species, this does not necessarily mean that these biodiversity hotspots have proper conservation area networks and that soricomorphs are adequately protected. As an example, Sarkar et al. (2008) assessed the Mesoamerica, Chocó, and Tropical Andes biodiversity hotspots and found that existing protected areas do not perform well in protecting the Red List species in these regions.
Another aspect highlighted by our study is that a considerable diversity of soricomorph taxa occurs only on islands, with a higher proportion of threatened taxa being endemic to islands. This result is important in terms of conservation because it shows that about one-third of the soricomorphs endemic to islands may be at serious risk of extinction if their remaining island habitats are altered. In this regard, particular attention should be paid to the conservation of mature forests on the Andaman Islands (for Crocidura andamanens, C. hispida, C. jenkinsi), on Sri Lanka (C. hikmiya, C. miya, Feroculus feroculus, Solisorex pearsoni, Suncus fellowesgordoni, Suncus 


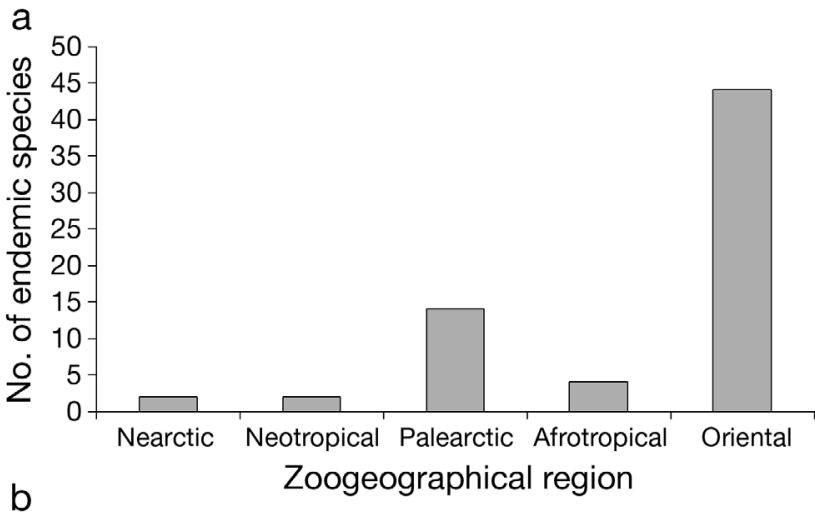

b

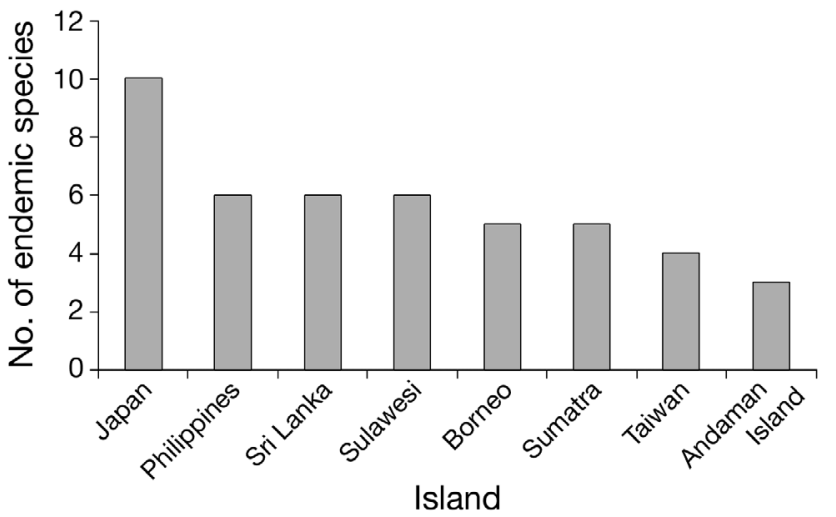

Fig. 3. Distribution of number of endemic soricomorph species by (a) zoogeographical region and (b) specific islands

zeylanicus), and Bioko (Myosorex eisentrauti, Sylvisorex isabellae).

In conclusion, although our study confirms that biodiversity hotspots covered the diversity of threatened soricomorphs quite satisfactorily (at least at the genus level), it should be noted that both mainland (Cameroon and Congo) and island (particularly the Andaman Islands, Sri Lanka, Bioko) forest ecosystems deserve particular attention from conservation organizations if soricomorphs are to be effectively maintained worldwide. In addition, the data available so far on soricomorphs are very preliminary and fragmentary (Stone 1995); hence, we strongly invite the scientific community to perform careful baseline studies on the ecology, taxonomy, and distribution of these mammals, particularly in the tropical regions, where species diversity is greater but less is known about the biology of these mammals.

\section{LITERATURE CITED}

Amori G, Gippoliti S (2000) What do mammalogists want to save? Ten years of mammalian conservation biology. Biodivers Conserv 9:785-793

Amori G, Luiselli L (2011) Small mammal community structures in West Africa: a meta-analysis using null models. Afr J Ecol doi:10.1111/j.1365-2028.2011.01274.x

Amori G, Gippoliti S, Helgen KM (2008) Diversity, distribution and conservation of endemic island rodents. Quat Int 182:6-15

Amori G, Chiozza F, Rondinini C, Luiselli L (2011a) Countrybased patterns of total species richness, endemicity, and threatened species richness in African rodents and insectivores. Biodivers Conserv 20:1225-1237

Amori G, Gippoliti S, Luiselli L (2011b) Do biodiversity hotspots match with rodent conservation hotspots? Biodivers Conserv doi:10.1007/s10531-011-0131-z

Brooks TM, Mittermeier RA, da Fonseca GAB, Gerlach J and others (2006) Global biodiversity conservation priorities. Science 313:58-61

Churchfield S (1990) The natural history of shrews. Christopher Helm, New York, NY

> Davis ALV, Philips TK (2009) Regional fragmentation of rain forest in west Africa and its effect on local dung beetle assemblage structure. Biotropica 41:215-220

Kareiva P, Marvier M (2003) Conserving biodiversity coldspots. Am Sci 91:344-351

Lamoreux JF, Morrison JC, Ricketts TH, Olson DM, Dinerstein E, McKnight MW, Shugart HH (2006) Global tests of biodiversity concordance and the importance of endemism. Nature 440:212-214

Mittermeier RA, Gil PR, Hoffmann M, Pilgrim J and others (2004) Hotspots revisited. CEMEX, Mexico City

Myers N (1998) Global biodiversity priorities and expanded conservation policies. In: Mace GM, Balmford A, Ginsberg JR (eds) Conservation in a changing world. Cambridge University Press, Cambridge, p 273-285

Myers N, Mittermaier RA, Mittermeier CG, da Fonseca GAB, Kent J (2000) Biodiversity hotspots for conservation priorities. Nature 403:853-858

Nowak RM (1999) Walker's mammals of the world, 6th edn. John Hopkins University Press, Baltimore, MD

Olson DM, Dinerstein E (1998) The Global 200: a representation approach to conserving the Earth's most biologically valuable ecoregions. Conserv Biol 12:502-515

Sala OE, Chapin FS III, Armesto JJ, Berlow E and others (2000) Global biodiversity scenarios for the year 2100. Science 287:1770-1774

Sarkar S, Sánchez-Cordero V, Londoño MC, Fuller T (2008) Systematic conservation assessment for the Mesoamerica, Chocó, and Tropical Andes biodiversity hotspots: a preliminary analysis. Biodivers Conserv 18:1793-1828

Stone D (1995). Eurasian insectivores and tree shrews. IUCN, Gland

Stuart W, Vivero Po JL, Spawls S, Shimelis A, Kelbessa E (2004) Ethiopian highlands. In: Mittermeier RA, Gil PR, Hoffmann M, Pilgrim J and others (eds) Hotspots revisited. CEMEX, Mexico City, p 262-273

Wilson DE, Reeder DR (2005) Mammal species of the world: a taxonomic and geographic reference, 3rd edn. John Hopkins University Press, Baltimore, MD

Wilson EO (1992) The diversity of life. WW Norton, New York, NY

Submitted: February 14, 2011; Accepted: August 23, 2011

Proofs received from author(s): October 27, 2011 